\title{
Measuring Extracellular Proton and Anionic Fluxes in Arabidopsis Pollen Tubes
}

\author{
Maria Teresa Portes* and José A. Feijó
}

Cell Biology and Molecular Genetics Department, University of Maryland, College Park, MD 20742, USA *For correspondence: mtportes@gmail.com

\begin{abstract}
[Abstract] The ion-selective vibrating probe has been used to detect and quantify the magnitude and direction of transmembrane fluxes of several ions in a wide range of biological systems. Inherently noninvasive, vibrating probes have been essential to access relevant electrophysiological parameters related to apical growth and morphogenesis in pollen tubes, a highly specialized cell where spatiotemporal tuning of ion dynamics is fundamental. Of relevance, crucial processes to the cell physiology of pollen tubes associated with protons and anions have been elucidated using vibrating probes, allowing the identification of diverse molecular players underlying and regulating their extracellular fluxes. The use of Arabidopsis thaliana as a genetic model system posed new challenges given their relatively small dimensions and difficult manipulation in vitro. Here, we describe protocol optimizations that made the use of the ion-selective vibrating probe in Arabidopsis pollen tubes feasible, ensuring consistent and reproducible data. Quantitative methods like this enabled characterizing phenotypes of ion transporter mutants, which are not directly detectable by evident morphological and reproductive defects, providing valuable insights into molecular and cellular mechanisms. The protocol for quantifying extracellular proton and anionic fluxes detailed here can be adjusted to other systems and species, while the sample preparation can be applied to correlated techniques, facilitating the research of pollen tube growth and development.
\end{abstract}

Keywords: Ionic fluxes, lon-selective vibrating probe, Non-invasive measurements, Phenotyping, Plant ion dynamics, Pollen tubes.

[Background] The relevance of bioelectricity and ion exchange for living cells is unquestionable, having a functional impact in a range of phenomena, from pattern formation, signaling and development to cancer and other diseases (Levin, 2014). Diverse techniques can be employed to detect action potentials, electric fields, extracellular electric currents, and ion fluxes. However, the assessment of their function in vivo requires non-invasive methods. Ideally, any biological system of interest should be studied with the minimum interference and under the most physiological condition possible. Such criteria are attained by the non-invasive ion-selective vibrating probe, which has been used for measuring multiple transmembrane ion fluxes in a wide variety of experimental systems, including Drosophila (Browne and O'Donnell, 2016), zebrafish (Guh et al., 2016), mouse skin (Sun et al., 2015), roots (He et al., 2015), Daphnia (Stensberg et al., 2014), C. elegans (Adlimoghaddam et al., 2014), etc. In pollen tubes, quantitative measurements of extracellular ion fluxes using the ion-selective vibrating probe have been fundamental in establishing the role of major ions (especially $\mathrm{Ca}^{2+}, \mathrm{H}^{+}, \mathrm{K}^{+}$, and anions) in apical growth. When used in association with reverse genetics and pharmacology targeting specific molecular 
players, these methods also allowed the identification of mutation effects and subtle phenotypes, such as through quantitative analysis of aberrant oscillatory behavior (Certal et al., 2008; Michard et al., 2011 and 2017; Portes et al., 2015; Wudick et al., 2018; Hoffmann et al., 2020).

The ion-selective vibrating probe is a technique designed to reduce the electric noise related with ionophore loaded, glass microelectrode probes, bringing the signal/noise ratio to levels compatible with the measurement of physiological extracellular electric/ionic currents, associated with single cells and other biological systems (Shipley and Feijó, 1999; Kunkel et al., 2006). The experimental setup comprises a customized microelectrode, front-loaded with ion-selective ionophore cocktails (LIX) that measures the voltage at two locations at close vicinity of the plasma membrane (with $\mu \mathrm{m}$ precision), so that most noise and drift induced bias is subtracted from the final output. In the described protocol/setup, two routines allow for extra quantitative precision: on one hand, the background reference is measured away from any cell and also subtracted from the final voltage difference for convection, thermal or ionic gradient compensation; on the other hand, background concentration of the measured ion is continuously monitored, allowing the quantitative normalization of the fluxes in real time using Fick's laws of diffusion.

The entire setup consists on an inverted microscope, nanometer 3-D positioners, and electrode impedance/capacitance correcting amplifier head stages, all placed inside a Faraday's cage to reduce environmental electrical noise. Individual probes are calibrated at the start and end of experiments by measuring their potential in 3 appropriate solutions with concentrations ranging 3 orders of magnitude, deemed usable if closer than $95 \%$ to the Nernstian potential over the reference solutions. All experimental output is generated by an external, variable-gain amplifier with an analog read-out, and fed through an A/D board into a dedicated computer. All data processing including the calibration procedure, probe quality control, 3-D stepper motor-driven probe positioning and movement (vibration) system is performed by the ASET software (Automated Scanning Electrode Techniques - Applicable Electronics). The spatial resolution is limited by the dimension of the probe tip, usually at 1-3 $\mu \mathrm{m}$ of diameter, allowing sampling of small and specific patches at the cell surface. Depending on the ion, flux resolution goes into the pmol $\mathrm{cm}^{-2} \mathrm{~s}^{-1}$ range (Shipley and Feijó, 1999; Kunkel et al., 2006).

The use of a first-generation wire/voltage detection vibrating probes led to the discovery of an electric field around pollen tubes, which have been proposed to be cells behaving as electrical dipoles (Weisenseel et al., 1975). While highlighting the importance of ion dynamics for pollen tube growth and development, these early measurements and interpretative models were affected by technical artefacts of these early wire electrode probes, namely the stirring induced by the hundreds of $\mathrm{Hz}$ vibration needed for the noise subtraction by lock-in amplifiers. Discussing these limitations (e.g., Shipley and Feijó, 1999) allowed to assess ionic derived currents, and the subsequent development of ion-specific glass microelectrodes methods. Besides measuring only a specific ion, instead of the electric current stemming from the sum of all ion transport over a given surface, it also vibrates at very low frequencies $(<0.5 \mathrm{~Hz})$ thus respecting the formation and stability of ion gradients over the cell surface during data acquisition. The resulting extensive effort led to the description of ion dynamics of pollen tubes in species as lily and tobacco (Feijó et al., 1999; Certal et al., 2008, Michard et al., 2008). The adoption of such 
species as study models was presumably related to the convenience of appropriated cell dimensions and high pollen germination and pollen tube growth rates, making them easy to obtain and experimentally manipulate in vitro. Despite the progress made using these species, the search for molecular mechanisms related to apical cell growth and sexual plant reproduction required the adoption of Arabidopsis thaliana as a genetic model. The application of these techniques to study ion dynamics and morphogenesis in Arabidopsis pollen is challenging due to the reduced dimensions of the flowers and difficulties in pollen manipulation in vitro. Besides these complications, the development of sophisticated molecular and genetic tools available for Arabidopsis required substantial protocol adaptations and optimizations, which were crucial for obtaining consistent data. Efforts to develop these protocols and approaches allowed the molecular identification and cellular localization of many ion transporters underlying the ion fluxes using ion-specific vibrating probes, together with the identification of key players involved in regulating ion homeostasis and morphogenesis in pollen tubes (reviewed in Michard et al., 2017).

The importance of protons and anions for pollen tube growth has been reported in diverse studies, where the presence of an intracellular gradient of these ions and the identification of the ion transporters promoting their movement was determined (Zonia et al., 2002; Michard et al., 2008; Gutermuth et al., 2013; Domingos et al., 2019). Of relevance, protons have been reported to enter mainly at the tip and generalized efflux occurring along the tube shank and pollen grain (Feijó et al., 1999; Certal et al., 2008; Hoffman et al., 2020). Anions, deemed as mostly chloride ( $\mathrm{Cl}-$ ) for being the only anion in the germination medium, have been shown to have an opposite flux direction, with influx along the pollen tube and grain and massive efflux at the tip (Zonia et al., 2002; Gutermuth et al., 2013; Domingos et al., 2019). Herein, a detailed description of the sample preparation and experimental protocol for measuring proton and anionic fluxes in pollen tubes with the ion-selective vibrating probe is presented.

Using quantitative techniques able to access measurable variables and parameters is complementary to genetics and imaging tools, offering a complete tool kit for characterizing mutant phenotypes. In this context, the use of ion-selective vibrating probes allowed the identification of phenotypes being otherwise undetectable, once diverse mutations do not display evident morphological and reproduction defects due to the high genetic redundancy and other compensatory mechanisms present in pollen tubes (Gutermuth et al., 2013; Wudick et al. 2018; Domingos et al., 2019). Experimental approaches coupling biophysical with molecular data are fundamental for an integrated and multidisciplinary investigation, contributing to better understand the mechanisms regulating intracellular cell physiology. The detailed protocol described below can be easily reproduced and adjusted to other systems, also enabling interested researchers to study pollen tube growth and development.

\section{Materials and Reagents}

1. Reference electrode - Dri-Ref (World Precision Instruments, catalog number: DRIREF-2)

2. Glass microfiber filters (Sigma-Aldrich, catalog number: WHA1820047)

3. Safe-Lock tubes, $1.5 \mathrm{ml}$ (Eppendorf, catalog number: 0030120086) 
4. Acrylic rectangular dish with a round chamber of $10 \mathrm{~mm}$ (custom-made) being the commercial equivalent the glass bottom dish (Cellvis, catalog number: D35-10-1-N)

5. Cover slip (Fisher Scientific, catalog number: 12-545)

6. Glass syringe (Hamilton, catalog number: 80600)

7. Silica gel (Sigma-Aldrich, catalog number: 13767)

8. Silver/silver chloride wire (Fisher Scientific, catalog number: AA41390G2)

9. Borosilicate glass capillaries (World Precision Instruments, catalog number: TW150-4)

10. N,N-Dimethyltrimethylsilylamine (Sigma-Aldrich, catalog number: 41716 )

11. Hydrogen ionophore II-cocktail A (Sigma-Aldrich, catalog number: 95297)

12. Chloride ionophore I-cocktail A (Sigma-Aldrich, catalog number: 99408)

13. Sucrose (Sigma-Aldrich, catalog number: S7903)

14. Potassium chloride (Sigma-Aldrich, catalog number: P9333)

15. Magnesium sulfate (Sigma-Aldrich, catalog number: 746452)

16. Boric acid (Sigma-Aldrich, catalog number: B6768)

17. Calcium chloride (Sigma-Aldrich, catalog number: C1016)

18. 4-(2-Hydroxyethyl)piperazine-1-ethanesulfonic acid (HEPES) (Sigma-Aldrich, catalog number: H3375)

19. Poly-L-lysine solution (Sigma-Aldrich, catalog number: P4707)

20. Agarose low gelling temperature (Sigma-Aldrich, catalog number: A9045)

21. Germination medium (see Recipes)

22. Stock solutions (see Recipes)

\section{Equipment}

1. $\mathrm{pH}$ meter

2. Vortex

3. Microwave

4. Oven

5. Desiccator

6. Fine needle-sharp tweezers (Sigma-Aldrich, catalog number: T4537)

7. Benchtop microcentrifuge (Eppendorf, model: 5415D)

8. Micropipette puller (Sutter Instrument Company, model: P-97)

9. Ion-Selective Vibrating Probe (System Applicable Electronics, model: SIET-Scanning Ionselective Electrode Technique)

10. Inverted microscope (Nikon, model: Eclipse TE300)

11. Microscope light power supply (Nikon, model: TE-PS100)

12. Camera (Andor, model: iXon3)

13. $60 x / 1.40$ Objective lens oil immersion (Nikon, model: Plan Apo) 


\section{Procedure}

The procedure description includes the detailed preparation of germination medium, experimental dishes, pollen collection, ion-selective microelectrodes, and saline bridges (only for anionic fluxes). The germination protocol was optimized from Boavida and McCormick (2007). Critically, the ionic background concentrations must be lowered as much as possible to minimize background noise when using the ion-selective vibrating probe technique. Thus, the concentration of all components of the germination medium was reduced aiming to reach a minimal, but functional, culture medium sufficient for pollen germination while increasing the signal-to-noise ratio.

A. Dishes preparation

Notes:

a. Keep stock solutions under $-20^{\circ} \mathrm{C}$.

b. Prepare fresh germination medium on the experimental day.

c. Due to the reduced dimensions of Arabidopsis pollen grains, the glass microelectrode can easily touch the glass bottom and break. To solve this problem, a thin layer of liquid agarose is placed over the glass, just enough to cover it. After drying, the agarose pad is coated with poly-L-lysine to increase the pollen adherence avoiding pollen tube floating. The ideal condition is having the pollen tube growing attached to the agarose pad facilitating the probe positioning. Pollen grains remain attached to the agar for few hours without dish agitation.

d. The poly-L-lysine should be properly removed to avoid toxicity.

1. Prepare fresh liquid germination medium from stock solutions.

2. Fix a cover slip at the bottom of the round chamber with $10 \mathrm{~mm}$ of diameter of the acrylic rectangular dish (not needed when using the commercial equivalent).

3. Melt $0.01 \%$ of low melting agarose in $1 \mathrm{ml}$ of germination medium in a safe-lock tube $(1.5 \mathrm{ml})$ for $30 \mathrm{~s}$ in the microwave.

4. Add $50 \mu \mathrm{l}$ to the bottom of the dish and remove the excessive liquid with a glass microfibre filter keeping only the volume sufficient to cover the glass surface but creating an agarose pad.

5. Add $50 \mu \mathrm{l}$ of poly-L-lysine on top of the agarose pad.

6. Wait 5 min.

7. Remove poly-L-lysine.

8. Wash twice with liquid medium to remove the excessive poly-L-lysine but still keeping the adhesive property.

B. Pollen collection and germination

Notes: 
a. Growing Arabidopsis plants preferentially under short-day photoperiod conditions increase pollen quality and stability, achieving high germination rates (> 90\%). Although, flowers grown under other photoperiods conditions can also be used.

b. Start to collect flowers from the $10^{\text {th }}$ silique. The first siliques have a low density of pollen and the germination rate is reduced.

c. Collect flowers until 1:00 pm, otherwise, the flowers start to close and pollen viability decreases;

d. Density is very important for Arabidopsis pollen germination. Collect 20-25 flowers per dish of $10 \mathrm{~mm}$ diameter well.

e. Resuspend pollen precipitate with a $200 \mu \mathrm{l}$ tip with the first millimeters cut to enlarge its aperture, as to avoid pollen grains damage.

f. To prepare the wet chamber use a Petri dish with a paper filter soaked in distilled water, to avoid medium evaporation during pollen germination.

1. Grow Arabidopsis plants until flowering (ecotype Columbia - Col-0) in growth chambers under short-day photoperiod conditions ( $12 \mathrm{~h}$ light/12 $\mathrm{h}$ dark cycle) at $22{ }^{\circ} \mathrm{C}$ with $70 \%$ humidity and light intensity of $\sim 100 \mu \mathrm{mol} \mathrm{m} \mathrm{m}^{-2} \mathrm{~s}^{-1}$ to improve pollen integrity and density.

2. Collect flowers immediately after anthesis (stage 13 - Smyth et al., 1990) using a thin tweezer and transfer to a $1.5 \mathrm{ml}$ tube (no more than 100 flowers per tube - use 20-25 flowers per dish).

3. Add $1 \mathrm{ml}$ of germination liquid medium.

4. Vortex at high speed $(\sim 2,500 \mathrm{rpm})$ for $30-40 \mathrm{~s}$.

5. Centrifuge at $1,600 \times g$ for $3 \mathrm{~min}$.

6. Remove flowers and supernatant.

7. Resuspend gently the pollen precipitate in $100 \mu \mathrm{l}$ of liquid medium.

8. Add one single drop $(\sim 25 \mu \mathrm{l})$ of the pollen precipitate in the experimental dish.

9. Add $200 \mu \mathrm{l}$ of liquid medium very slowly to keep pollen grains attached to the agarose pad.

10. Incubate the dishes in a wet chamber at $22{ }^{\circ} \mathrm{C}$, preferentially in the dark.

11. After $2-3 \mathrm{~h}$ growing pollen tubes with $\geq 200 \mu \mathrm{m}$ of length can be assayed.

The procedure is illustrated in Figure 1.

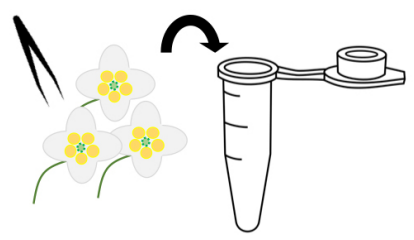

Collect newly opened flowers and transfer to a tube

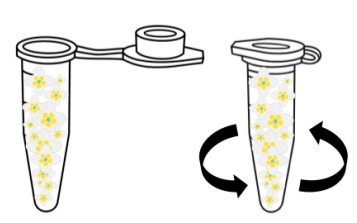

Vortex and centrifuge at $1600 \mathrm{~g}$ for $3 \mathrm{~min}$

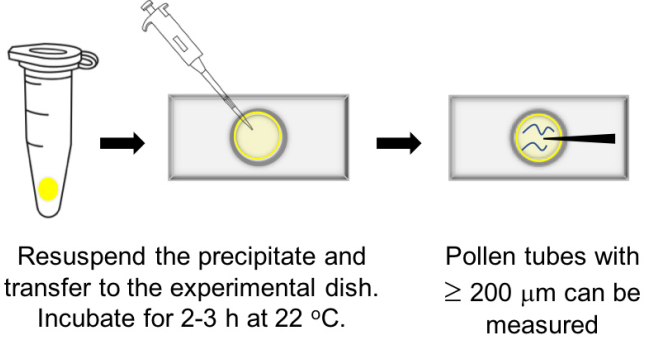

Figure 1. Schematic representation of flower collection and sample preparation steps.

The illustration shows how to obtain pollen from flowers, followed by resuspension and 
incubation of pollen grains to promote pollen germination. Finally, extracellular ionic measurements can be performed in sufficiently long Arabidopsis pollen tubes.

C. Glass micropipette silanization and ion-selective probe preparation Notes:

a. Optimizing the puller parameters to obtain ion-selective probes according to the desired pipette configuration. The tip aperture should have $1-3 \mu \mathrm{m}$ of diameter for measuring both protons and anions, while the taper is slightly longer for anions to decrease ionophore leakage (see Shipley and Feijó, 1999 for reference).

b. Ionophores are commercialized by different brands, being purity and quality important factors that can drastically vary according to the manufacturer, having impact on the Nernst values measured by the electrode. Furthermore, discrepant Nernst values could be an indication that the ionophore is old or contaminated.

1. Pulling proton- and anionic-specific micropipettes.

2. Place the micropipettes in a wire net or Teflon chamber that enables air circulation (Figure 2).

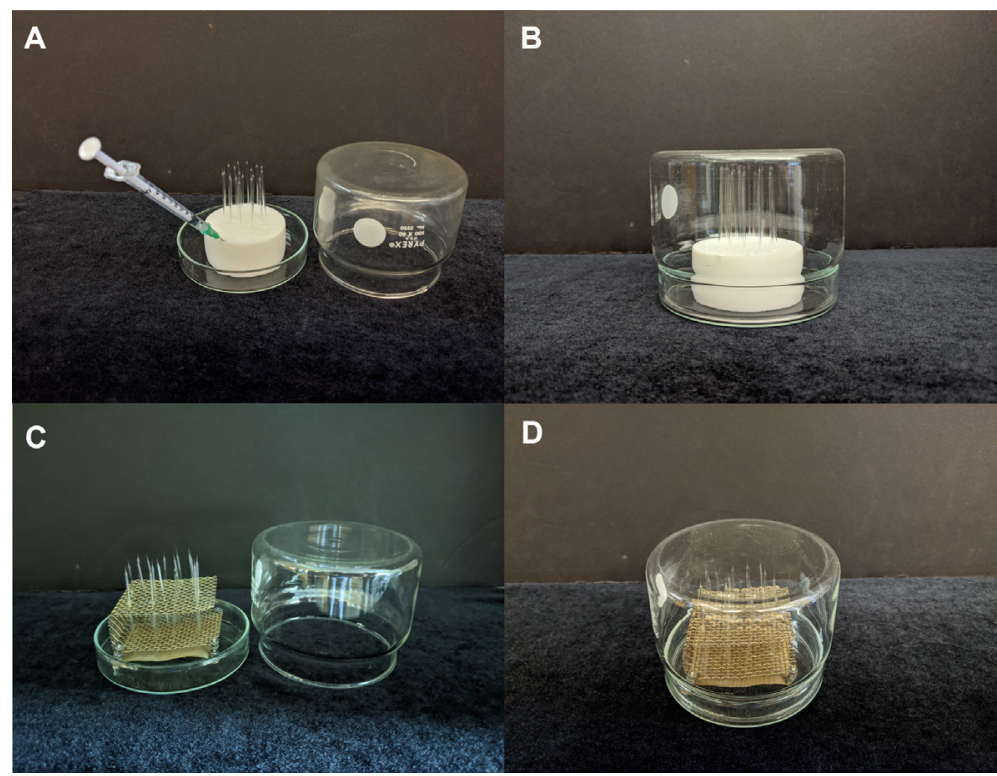

Figure 2. Glass micropipettes silanization using a wire net or a Teflon chamber. A. Micropipettes placed on a Teflon chamber for silanization. Syringe indicates the aperture where the $\mathrm{N}, \mathrm{N}$-Dimethyltrimethylsilylamine is applied. B. Closed chamber ready for the silanization procedure. C. Micropipettes placed on a wire net for silanization. D. Closed chamber ready for the silanization procedure.

3. Keep the micropipettes overnight in the oven at $210^{\circ} \mathrm{C}$.

4. Add $90 \mu \mathrm{l}$ of $\mathrm{N}, \mathrm{N}$-dimethyltrimethylethylsilamine with a glass syringe.

5. Wait 30 min. 
6. Let the micropipettes cool down inside the oven.

7. Store the silanized micropipettes in a desiccator with silica gel.

8. Using a syringe, backfill the microelectrode with a $20-25 \mathrm{~mm}$ column of $40 \mathrm{mM} \mathrm{KH}_{2} \mathrm{PO}_{4} / 15 \mathrm{mM}$ $\mathrm{KCl}, \mathrm{pH} 7.5$, and front-loaded with a $\sim 25 \mu \mathrm{m}$ column of the hydrogen or chloride ionophore cocktail.

D. Ion-selective probe calibration

Notes:

a. Calibration solutions used for $H^{+}$flux measurements are $p H$ 5, 6, and 7 and 0.1, 1, and $10 \mathrm{mM}$ $\mathrm{KCl}$ for anionic measurements.

b. As the chloride ionophore detects other anions in addition to $\mathrm{Cl}^{-}$, the estimates are referred to anionic fluxes instead of chloride fluxes.

c. A stable $\mathrm{H}^{+}$-electrode can be used for 4-5 $h$ of measurements; it is recommended to check its stability by measuring one of the calibration solutions every $2 h$.

$d$. The anionic-electrode should be prepared and stabilized for $\sim 2 h$ before calibration. Electrode stabilization consists of keeping the electrode in the $0.1 \mathrm{mM} \mathrm{KCl}$ calibration solution. Moreover, after stabilization and calibration, the anionic-electrode can be used only for $1 \mathrm{~h}$ for ensuring reliable measurements.

e. The reference electrode (Dri-Ref) should be kept in $3 \mathrm{M} \mathrm{KCl}$ and properly rinsed with water before use.

f. The silver/silver chloride wire connected to the pipette holder has to go through the electrochloridizing process before use. The process consists of removing any coating residue from the wire with a small piece of thin sandpaper. After that, a $9 \mathrm{~V}$ battery is connected to a dual crocodile clip cable, where in one end has a thick silver wire attached and the other end has the metal pin of the pipette holder. Both wires ends must be immersed at the same time in a solution of $1 \mathrm{M}$ $\mathrm{KCl}$ for a few seconds. When the silver wire of the pipette holder becomes darker, it is ready to use.

1. Connect the microelectrode at the holder ensuring the contact of silver/silver chloride wire with the filling solution.

2. Place the microelectrode in the 3 calibration solutions recording the voltage values individually, estimating the adequate Nernst potential fitting.

3. After calibration, place the microelectrode in the experimental dish.

4. Close the circuit by inserting the dry reference electrode also into the dish (Figure 3). 


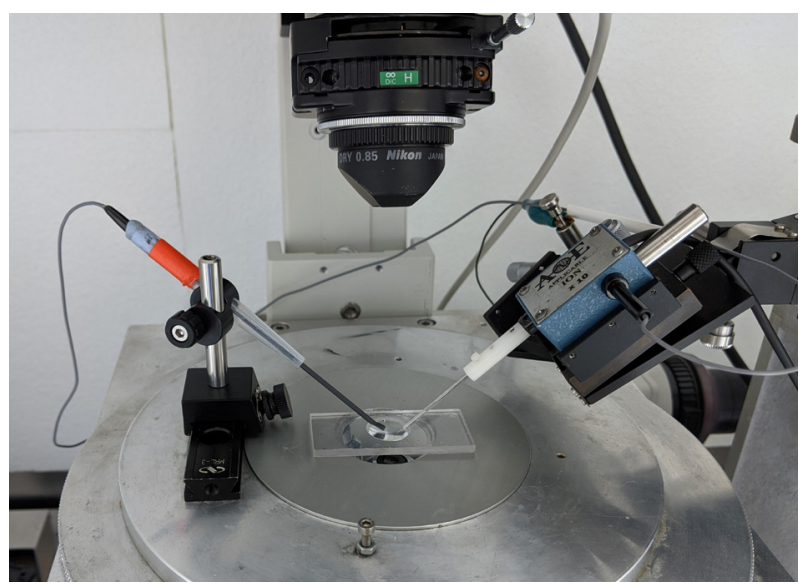

Figure 3. Experimental setup and sample dish. Ion-selective vibrating probe setup consisting of the reference electrode (left), experimental dish and the ion-selective microelectrode connected to pipette holder.

5. Position the microelectrode close enough to the plasma membrane of the pollen tube but avoid touching it (Figure 4, Videos 1 and 2).

6. Measure the background reference in a region $>500 \mu \mathrm{m}$ away from any pollen grain or pollen tube.

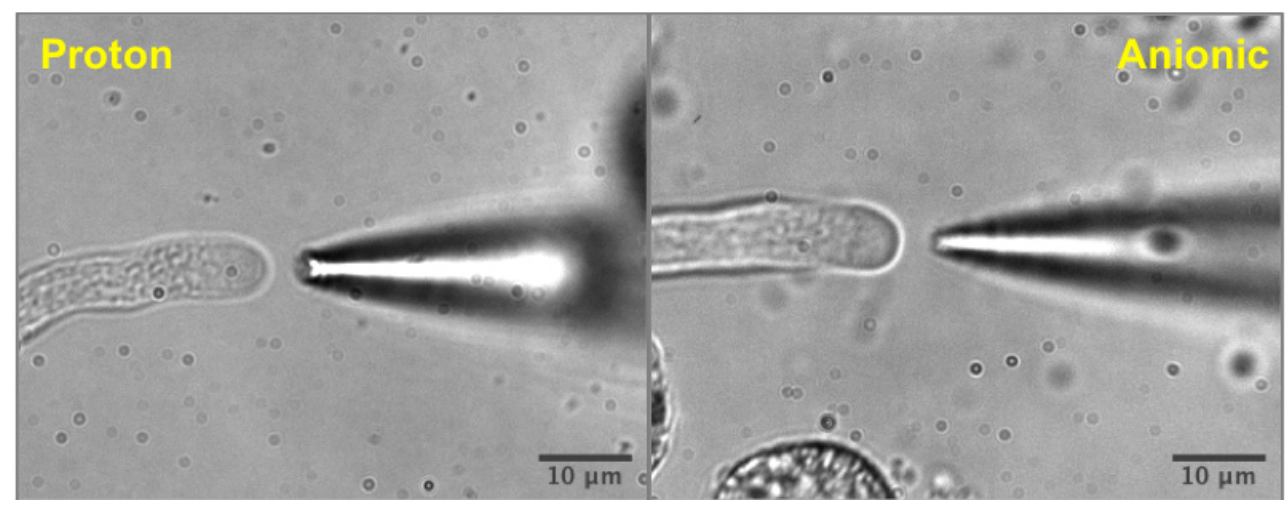

Figure 4. Proton- and anionic-electrodes. Examples of a proton-specific (left) and anionicspecific electrode (right) placed in front of the tip of Arabidopsis pollen tubes. Anionic-specific electrodes have a longer taper and slightly smaller tip aperture. 


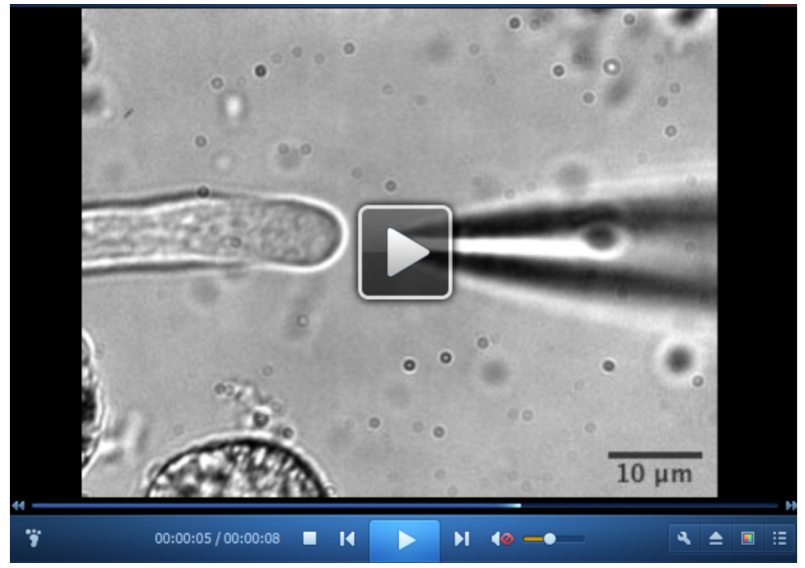

Video 1. Timelapse from extracellular proton measurement at the tip of Arabidopsis pollen tube. Acquisition of 100 frames with $4 \mathrm{~s}$ interval.

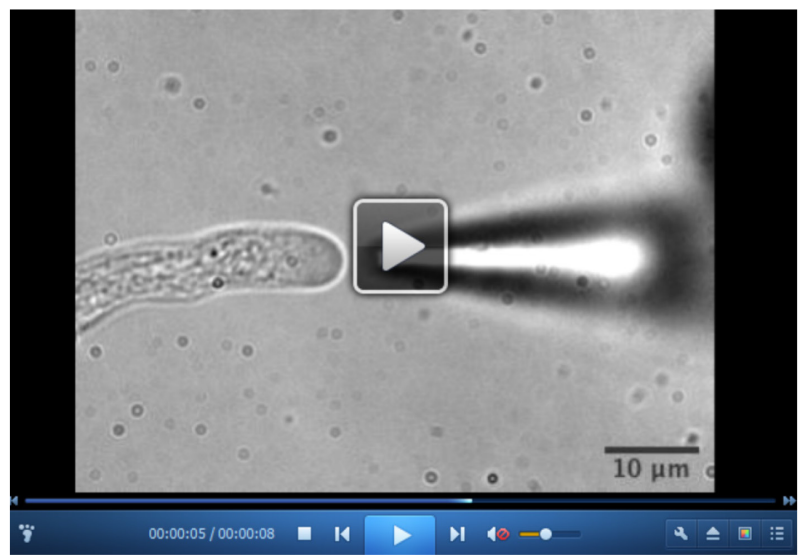

Video 2. Timelapse from extracellular anionic measurements at the tip of Arabidopsis pollen tube. Acquisition of 100 frames with $4 \mathrm{~s}$ interval.

\section{E. Data processing}

1. Save the output data from the ASET software.

2. Use data from ASET output to calculate fluxes as set up on the Excel file provided. The spreadsheet has as input the following constants: Electrode excursion, ionophore efficiency, diffusion coefficient, slope, and intercept of the calibration curve. In the cells below the constants one should input the ASET 'Background' and 'Voltage difference' measurements that will produce an output in the columns to the right of the calculation spreadsheet. One should then select all rows of the output and drag it down until it matches the end of the input rows.

The spreadsheet subtracts the background reference values from the voltage differential recordings and calculates ion fluxes using Fick's law for protons using diffusion coefficient 9.37 $\times 10^{-5} \mathrm{~cm}^{-2} \mathrm{~s}^{-1}$ efficiency 1 ; and for chloride diffusion coefficient $-2.03 \times 10^{-5} \mathrm{~cm}^{-2} \mathrm{~s}^{-1}$ and efficiency 0.5 .

\section{F. Salt bridges (used only for anionic flux measurements)}




\section{bĭ̈-protocol

Note: Salt bridges are used for isolating the biological sample from the reference electrode, avoiding the germination medium contamination with high concentrations of $\mathrm{KCl}$, leaking from the reference electrode. Otherwise, the chloride concentration would substantially increase in the culture medium altering the signal-to-noise ratio, generating a source of error in these estimates. The salt bridge establishes the electrical contact between the two dishes but keeps the reference electrode isolated from the biological sample avoiding contamination and inaccuracies related to the ionic concentration fluctuation during the measurement.

1. Create a bridge by heating the center of a glass capillary and folding it using two forceps to create a bridge with a "V" format.

2. Fill the bridge with a jellified solution of germination liquid medium with $0.01 \%$ of low melting point agarose.

3. Place one part of the bridge in the dish where the pollen tubes are growing (where the ionselective probe is placed) and the other part of the bridge is inserted in the other dish filled only with liquid medium (where the reference electrode is placed) (Figure 5).

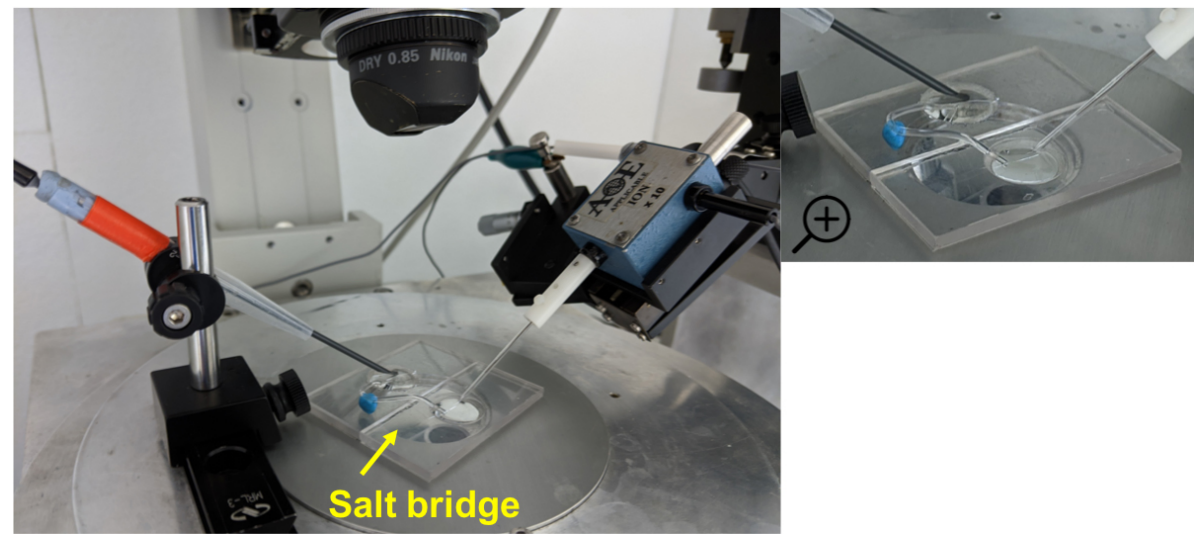

Figure 5. Salt bridge for anionic fluxes measurements. The anion-specific electrode is placed into the experimental dish while the reference electrode is placed in another dish filled with germination medium. The salt bridge connects both dishes closing the measuring circuit.

The setup is zoomed in on the right.

\section{$\underline{\text { Recipes }}$}

1. Germination medium

$\begin{array}{ll}500 \mu \mathrm{M} & \mathrm{KCl} \\ 500 \mu \mathrm{M} & \mathrm{CaCl}_{2} \\ 125 \mu \mathrm{M} & \mathrm{MgSO}_{4} \\ 0.005 \%(\mathrm{w} / \mathrm{v}) & \mathrm{H}_{3} \mathrm{BO}_{3} \\ 125 \mu \mathrm{M} & \mathrm{HEPES} \\ 16 \%(\mathrm{w} / \mathrm{v}) & \text { sucrose }\end{array}$


Adjust $\mathrm{pH}$ to 7.5 with $\mathrm{NaOH}$

2. Stock solutions

Stock $\mathrm{KCl} \quad 100 \mathrm{mM}$

Stock $\mathrm{CaCl}_{2} \quad 100 \mathrm{mM}$

Stock $\mathrm{MgSO}_{4} \quad 100 \mathrm{mM}$

Stock $\mathrm{H}_{3} \mathrm{BO}_{3} \quad 1 \%$

Stock HEPES $\quad 100 \mathrm{mM}$

\section{Acknowledgments}

J.F. lab was supported by the National Science Foundation grants (MCB 1616437/2016 and 1930165/2019) and the University of Maryland. We thank Custódio de Oliveira Nunes and Michael A. Lizzio for the photos.

\section{Competing interests}

There are no conflicts of interest or competing interest.

\section{References}

1. Adlimoghaddam, A., Weihrauch, D. and O'Donnell, M. J. (2014). Localization of $\mathrm{K}^{+}, \mathrm{H}^{+}, \mathrm{Na}^{+}$and $\mathrm{Ca}^{2+}$ fluxes to the excretory pore in Caenorhabditis elegans: application of scanning ionselective microelectrodes. J Exp Biol. 217: 4119-4122.

2. Boavida, L. C. and McCormick, S. (2007). Temperature as a determinant factor for increased and reproducible in vitro pollen germination in Arabidopsis thaliana. Plant J 52: 570-82.

3. Browne, A. and O'Donnell, M.J. (2016). Segment-specific $\mathrm{Ca}^{2+}$ transport by isolated Malpighian tubules of Drosophila melanogaster: A comparison of larval and adult stages. $J$ Insect Physiol 87: 1-11.

4. Certal, A. C., Almeida, R. B., Carvalho, L. M., Wong, E., Moreno, N., Michard, E., Carneiro, J., Rodriguez-Leon, J., Wu, H. M., Cheung, A. Y. and Feijo, J. A. (2008). Exclusion of a proton ATPase from the apical membrane is associated with cell polarity and tip growth in Nicotiana tabacum pollen tubes. Plant Cell 20(3): 614-634.

5. Domingos, P., Dias, P., Tavares, B., Portes, M. T., Wudick,, M. M., Konrad, K., Gilliham, M., Bicho, A. and Feijó, J. A. (2019). Molecular and electrophysiological characterization of anion transport in Arabidopsis thaliana pollen reveals regulatory roles for $\mathrm{pH}, \mathrm{Ca}^{2+}$ and $\mathrm{GABA}$. New Phytol 223: 1353-1371.

6. Feijó, J. A., Sainhas, J., Hackett, G. R., Kunkel, J. G. and Hepler, P. K. (1999). Growing pollen tubes possess a constitutive alkaline band in the clear zone and a growth-dependent acidic tip. J Cell Biol 144: 483-496. 
Please cite this article as: Portes and Feijó, (2021). Measuring Extracellular Proton and Anionic Fluxes in Arabidopsis Pollen Tubes,Bio-protocol 11 (3):

7. Guh, Y. J., Yang, C. Y., Liu, S. T., Huang, C. J. and Hwang, P. P. (2016). Oestrogen-related receptor $\alpha$ is required for transepithelial $\mathrm{H}^{+}$secretion in zebrafish. Proc Biol Sci 283(1825): 20152582.

8. Gutermuth, T., Lassig R., Portes M. T., Maierhofer T., Romeis, T., Borst J., Hedrich R., Feijó J. A. and Konrad K. R. (2013). Pollen tube growth regulation by free anions depends on the interaction between the anion channel SLAH3 and calcium-dependent protein kinases CPK2 and CPK20. Plant Cell 25: 4525-4543.

9. He, Y., Wu, J., Lv, B., Li, J., Gao, Z., Xu, W., Baluska, F., Shi, W., Shaw, P. C. and Zhang, J. (2015). Involvement of 14-3-3 protein GRF9 in root growth and response under polyethylene glycol-induced water stress. J Exp Bot 66(8): 2271-2281.

10. Hoffmann, R. D., Portes, M. T., Olsen, L. I., Damineli, D. S. C., Jesper, M. H., Pedersen, T., Lima, P. T. Campos, C., Feijó, J. A. and Palmgren. M. (2020). Plasma membrane $\mathrm{H}^{+}-A T P a s e s$ sustain pollen tube growth and fertilization. Nat Comm 11(1): 2395.

11. Kunkel, J. G., Cordeiro, S., Xu, J., Shipley, A. M. and Feijó, J. A. (2006). Use of non-invasive ion-selective microelectrode techniques for the study of plant development. In: Volkov, V. (Ed.). Plant electrophysiology-Theory and methods. Springer, Berlin, pp 109-137.

12. Levin, M. (2014). Molecular bioelectricity: how endogenous voltage potentials control cell behavior and instruct pattern regulation in vivo. Mol Biol Cell 25(24): 3835-3850.

13. Michard, E., Lima, P. T., Borges, F., Silva, A. C., Portes, M. T., Carvalho, J. E., Gilliham, M., Liu L-H., Obermeyer, G. and Feijó J. A. (2011). Glutamate receptor-like genes form $\mathrm{Ca}^{2+}$ channels in pollen tubes and are regulated by pistil D-serine. Science 332: 434-437.

14. Michard, E., Simon, A. A., Tavares, B., Wudick, M. M. and Feijó, J. A. (2017). Signaling with ions: The keystone for apical cell growth and morphogenesis in pollen tubes. Plant Physiol 173: 91-111.

15. Michard, E., Dias, P. and Feijó, J. A. (2008). Tobacco pollen tubes as cellular models for ion dynamics: improved spatial and temporal resolution of extracellular flux and free cytosolic concentration of calcium and protons using pHluorin and YC3.1 CaMeleon. Sex Plant Reprod 21: 169-181.

16. Portes, M. T.,Damineli, D. S. C., Moreno, N., Colaço, R., Costa, S. and Feijó, J. A. (2015). The pollen tube oscillator: Integrating biophysics and biochemistry into cellular growth and morphogenesis. In: Mancuso, S. and Shabala, S. (Eds.). Rhythms in plants: dynamic responses in a dynamic environment. Springer, Cham, pp. 121-156.

17. Shipley, A. M. and Feijó, J. A. (1999). The use of the vibrating probe technique to study steady extracellular currents during pollen germination and tube growth. In: Cresti M, Cai G, Moscatelli A (eds) Fertilization in higher plants: molecular and cytological aspects. Springer, Berlin, pp 235252.

18. Smyth, D. R., Bowman, J. L. and Meyerowitz, E. M. (1990). Early flower development in Arabidopsis. Plant Cell 2(8): 755-767. 
19. Stensberg, M. C., Zeitchek, M. A., Inn, K., McLamore, E. S., Porterfield D. M. and Sepúlveda, M. S. (2014). Comparative study of non-invasive methods for assessing Daphnia magna embryo toxicity. Environ Sci Pollut Res 21: 10803-10814.

20. Sun, Z., Yue, J., and Zhang, Q. (2015). lonic components of wound current at mouse skin incisional wounds. Eur J Med Res 1(1): 3-7.

21. Weisenseel, M. H., Nuccitelli, R. and Jaffe, L. F. (1975). Large electrical currents traverse growing pollen tubes. J Cell Biol 66(3): 556-567.

22. Wudick, M. M., Portes M. T., Michard, E., Rosas-Santiago, P., Lizzio, M. A., Nunes, C. O., Campos, C., Damineli, D. S. C., Carvalho, J. C., Lima, P. T., Pantoja, O. and Feijó, J. A. (2018). CORNICHON sorting and regulation of GLR channels underlie pollen tube $\mathrm{Ca}^{2+}$ homeostasis. Science 360(6388): 533-536.

23. Zonia, L., Cordeiro, S., Tupý, J. and Feijó, J. A. (2002). Oscillatory chloride efflux at the pollen tube apex has a role in growth and cell volume regulation and is targeted by inositol 3,4,5,6tetrakisphosphate. Plant Cell 14(9): 2233-49. 